\title{
Isoalantolactone inhibits the migration and invasion of human breast cancer MDA-MB-231 cells via suppression of the p38 MAPK/NF-кB signaling pathway
}

\author{
JING WANG ${ }^{1,2}$, LI CUI $^{1,2}$, LIANG FENG ${ }^{1,2}$, ZHENHAI ZHANG $^{1,2}$, JIE SONG $^{1,2}$, \\ DAN LIU ${ }^{1,2}$ and XIAOBIN JIA ${ }^{1,2}$
}

\begin{abstract}
${ }^{1}$ Affiliated Hospital of Integrated Traditional Chinese and Western Medicine, Nanjing University of Chinese Medicine, Nanjing, Jiangsu 210028; ${ }^{2}$ Key Laboratory of New Drug Delivery System of Chinese Materia Medica, Jiangsu Province Academy of Chinese Medicine, Nanjing, Jiangsu 210028, P.R. China
\end{abstract}

Received January 19, 2016; Accepted March 23, 2016

DOI: $10.3892 /$ or.2016.4954

\begin{abstract}
Isoalantolactone is a bioactive sesquiterpene lactone isolated from the flowering plant Inula helenium L. This study was conducted to assess the anti-migratory and anti-invasive activities of isoalantolactone in MDA-MB-231 cells, and to explore the underlying mechanisms. Wound-healing and Transwell chambers assays demonstrated that isoalantolactone inhibited the adhesion, migration and invasion of MDA-MB231 cells. The activity and expression of MMP-2 and MMP-9 were downregulated by isoalantolactone in a dose-dependent manner. Additionally, isoalantolactone markedly decreased the p-p38 MAPK level, whereas no significant change in p-ERK $1 / 2$ and p-JNK1/2 was noted. The downregulation of MMP-2 and MMP-9 protein expression and suppression of in vitro invasion might be associated with the blockade of p38 MAPK activation. Furthermore, isoalantolactone blocked the translocation of NF- $\mathrm{KB}$ p 65 from the cytoplasm into the nucleus. These results revealed that isoalantolactone inhibited the adhesion, migration and invasion of MDA-MB-231 cells via suppression of the $\mathrm{p} 38 \mathrm{MAPK} / \mathrm{NF}-\mathrm{\kappa B}$ signaling pathway, and isoalantolactone might be an alternative treatment for breast cancer.
\end{abstract}

\section{Introduction}

Breast cancer is one of the most aggressive cancers among females worldwide (1). Radiotherapy, surgery and molecular-targeted drug therapies have been applied in the treatment

Correspondence to: Dr Li Cui or Dr Xiaobin Jia, Key Laboratory of New Drug Delivery Systems of Chinese Materia Medica, Jiangsu Provincial Academy of Chinese Medicine, 100 Shizi Street, Hongshan Road, Nanjing, Jiangsu 210028, P.R. China

E-mail: cuili2008516@126.com

E-mail: xiaobinjia_nj@126.com

Key words: isoalantolactone, migration, invasion, p38 MAPK, nuclear factor- $\kappa \mathrm{B}$ of breast cancer. However, these conventional therapies cause serious side-effects, deeply affecting the quality of life of patients (2). Therefore, there is an urgent need to search for safe and effective drugs for the treatment of breast cancer.

Growing evidence suggests that matrix metalloproteinases (MMPs) play an important role in cell invasion and metastasis, since they essentially degrade the extracellular matrix (ECM) (3). Among the MMPs, the protein level and activity of MMP-2 (gelatinase A, $72 \mathrm{kDa}$ ) and MMP-9 (gelatinase B, $92 \mathrm{kDa}$ ) are frequently increased in metastatic carcinomas, including colon, lung and breast (4-6). In cancer cells, increased MMP-2 and MMP-9 activity and expression require constitutive mitogen-activated protein kinase (MAPK) activity, which includes p38 MAPK, c-Jun N-terminal kinase (JNK) and extracellular signal-regulated kinase (ERK) (7-10). The expression of MMP-2 and MMP-9 also depends on the activity of activator protein-1 (AP-1) and nuclear factor- $\mathrm{\kappa B}$ (NF- $\kappa \mathrm{B})$, which are the downstream factors of the MAPK signaling pathway (11).

Isoalantolactone (Fig. 1A), a sesquiterpene lactone isolated from the flowering plant Inula helenium L., has been shown to hold various pharmacological activities, including antitrypanosomal, anti-apoptosis, anti-microbial activities (12-15). It has also been reported that isoalantolactone possesses anticancer activity in several types of cancer cells, such as neck squamous cell carcinoma, prostate cancer and gastric adenocarcinoma cells (16-18). However, the ability of isoalantolactone to inhibit the migration and invasion of metastatic breast cancer cells remains unknown, and the underlying mechanism responsible for these effects is unclear. In this study, we investigated the effects of isoalantolactone on the viability, adhesion, migration and invasion of a highly metastatic human breast cancer cell line MDA-MB-231, and further explored the underlying molecular mechanism of action.

\section{Materials and methods}

Chemicals and drugs. Isoalantolactone (purity, $\geq 98 \%$ ) was obtained from Sigma Chemical Co. (St. Louis, MO, USA), and it was dissolved in dimethyl sulfoxide (DMSO) as primary 
stock solution. The solution was stored at $-20^{\circ} \mathrm{C}$, and diluted with medium for further study. During the process of the experiment, the final concentration of DMSO was $<0.5 \%$. The primary antibodies for MMP-2, MMP-9, p38 MAPK, p-p38 MAPK, JNK, p-JNK, p-ERK, ERK, NF-кB p65 and $\beta$-actin were purchased from Santa Cruz Biotechnology (Santa Cruz, CA, USA). Secondary antibodies were from Amersham Biosciences (Freiburg, Germany). L-15 medium, fetal bovine serum (FBS), 3-(4,5-dimethylthiazol-2-yl)-2,5-diphenyltetrazolium bromide (MTT), trypsin, Tween-20, and sodium dodecyl sulfate (SDS) were purchased from Sigma-Aldrich. Matrigel was purchased from BD Biosciences (San Jose, CA, USA). Other reagents were obtained from commercial sources.

Cell culture. Human breast cancer cell line, MDA-MB-231, was purchased from the Cell Bank of the Shanghai Institute of Cell Biology (Shanghai, China). The cells were maintained in L-15 medium supplemented with $10 \%$ FBS, $100 \mathrm{U} / \mathrm{ml}$ penicillin and $100 \mu \mathrm{g} / \mathrm{ml}$ streptomycin at $37^{\circ} \mathrm{C}$ with $5 \% \mathrm{CO}_{2}$ in a humidified atmosphere.

Cell viability assay. Cell viability was determined by MTT assay (19). MDA-MB-231 cells were seeded at a density of $1 \times 10^{4}$ cells/well $(100 \mu \mathrm{l})$ and treated with various concentrations of isoalantolactone for $24 \mathrm{~h}$. Subsequently, the medium was removed and $10 \mu \mathrm{l}$ of MTT solution $(5 \mathrm{mg} / \mathrm{ml})$ was added to each well for an additional $4 \mathrm{~h}$. At the end of the incubation, $100 \mu \mathrm{l}$ DMSO was added for $10 \mathrm{~min}$ after the removal of medium, owing to the fact that the number of viable cells was directly proportional to the production of formazan, which can be solubilized by DMSO. The optimal density (OD) was measured at $570 \mathrm{~nm}$ on a SpectraMax 190 (Molecular Devices, Sunnyvale, CA, USA). All the experiments were repeated three times.

Cell adhesion assay. After being incubated with different concentrations of isoalantolactone at $37^{\circ} \mathrm{C}$ for $24 \mathrm{~h}$, MDA-MB-231 cells were digested by $0.25 \%$ trypsin and centrifuged at $2,000 \mathrm{x} \mathrm{g}$ for $2 \mathrm{~min}$. Then, the cells were seeded into a 96 -well plate $\left(1 \times 10^{4}\right.$ cells/well), each of which was coated with $30 \mu \mathrm{g}$ Matrigel (BD Biosciences) to form a basement membrane. At the end of the incubation for $1 \mathrm{~h}$, the medium was discarded, and the cells were washed with $100 \mu \mathrm{l}$ phosphate-buffered saline (PBS) for three times to remove the non-adherent cells. Subsequently, MTT $(5 \mathrm{mg} / \mathrm{ml})$ in L-15 medium was added into each well and the attached cells were incubated at $37^{\circ} \mathrm{C}$ for $4 \mathrm{~h}$. DMSO $(100 \mu \mathrm{l})$ was added into each well, and shaken for $10 \mathrm{~min}$. The following processes were performed using the same method as for measurement of cell viability. The cell adhesion rate was calculated using the formula: Cell adhesion rate $=(\mathrm{OD}$ drug$/ \mathrm{OD}$ blank $) \times 100 \%$.

Wound-healing assay. The wound-healing assay was performed as described previously (20). MDA-MB-231 cells were seeded into 12 -well plates $\left(1 \times 10^{5}\right.$ cells/well $)$ in L-15 medium supplement. Cells were grown to nearly $80 \%$ confluency overnight and were then scratched using a $200-\mu 1$ pipette tip. The suspended cells were washed away and the wounded cell monolayer was incubated in FBS-free medium with isoalantolactone and/or other agents for $24 \mathrm{~h}$. The cells were visualized under a IX73 microscope (Olympus, Tokyo, Japan) at 0 and $24 \mathrm{~h}$. After treatment, the relative wound area was analyzed using Image-Pro Plus 6.0 software (Media Cybernetics, Rockville, MD, USA). Three independent experiments were performed.

Cell invasion assay. The effect of isoalantolactone on the invasion of MDA-MB-231 cells was evaluated in vitro as described previously (21). Matrigel (5 mg/ml; Becton Dickinson, Bedford, MA, USA) was applied to $8-\mathrm{mm}$ pore size polycarbonate membrane filters of a Transwell chamber (Corning Costar, Cambridge, MA, USA) at $37^{\circ} \mathrm{C}$ for $1 \mathrm{~h}$. MDA-MB-231 cells $\left(1 \times 10^{5}\right.$ cells/well) were seeded on the upper part of the chamber. The bottom chamber was filled with $500 \mu \mathrm{l}$ of L-15 medium containing $10 \%$ FBS. After treatment with isoalantolactone and/or other agents for $24 \mathrm{~h}$, the cells penetrating through the Matrigel and migrating to the lower chamber were fixed with paraformaldehyde, and stained with $0.1 \%$ crystal violet, and photographed. Invasion of the MDA-MB-231 cells was defined in terms of the total number of invasive cells in five randomly selected fields.

RNA extraction and quantitative real-time PCR. After being treated with isoalantolactone and/or other agents, cellular RNA was extracted and reversely transcripted to determine the expression of MMP-2 and MMP-9. According to the manufacturer's protocols (Invitrogen, Carlsbad, CA, USA), total RNA was isolated from the MDA-MB-231 cells using TRIzol reagent (Invitrogen Life Technologies, Waltham, MA, USA), followed by DNase I digestion (Thermo Fisher Scientific, Waltham, MA, USA). Complementary DNA was synthesized using PrimeScript ${ }^{\mathrm{TM}}$ RT reagent kit (Fermentas, Germany).

Real-time PCR was performed to detect mRNA levels, along with the ABI PRISM 7900 sequence detection system (Applied Biosystems, Foster City, CA, USA). Glyceraldehyde 3 -phosphate dehydrogenase $(\mathrm{GAPDH})$ was chosen as an internal standard. Primer sequences $\left(5^{\prime} \rightarrow 3^{\prime}\right)$ and probe numbers were as follows: MMP-2 forward, TTG ACG GTA AGG ACG GAC TC and reverse, CAT ACT TCA CAC GGA CCA CTT G; MMP-9 forward, TTC CAG TAC CAA GAC AAA GCC and reverse, CAC GGT TGA AGC AAA GAA GG; and GAPDH forward, CAC CCA CTC CTC CAC CTT TGA C and reverse, GCA ACT GTG AGG AGG GGA GAT T. Results were normalized to the GAPDH expression level, and relative quantitation of MMP-2 and MMP-9 was analyzed using the comparative $2^{-\Delta \Delta C t}$ method.

Preparation of cytosolic and nuclear fractions. MDA-MB231 cells were treated with or without isoalantolactone for $24 \mathrm{~h}$. Then, the cells were washed three times in ice-cold PBS followed by lysis in 1\% Triton X-100 lysis buffer $(10 \mathrm{mM}$ HEPES, pH 7.9, $10 \mathrm{mM} \mathrm{KCl,} 0.1 \mathrm{mM}$ EDTA, 0.5\% Nonidet P-40, $1 \mathrm{mM}$ dithiothreitol, $0.5 \mathrm{mM}$ phenylmethylsulfonyl fluoride). The cytosolic fraction was collected and centrifuged at $14,000 \mathrm{x}$ g for $15 \mathrm{~min}$ at $4^{\circ} \mathrm{C}$, and the nuclear pellets were resuspended in $1 \%$ Triton X-100 lysis buffer containing $400 \mathrm{mM}$ $\mathrm{NaCl}$. The nuclear extract was recovered after centrifugation at $14,000 \mathrm{xg}$ for $15 \mathrm{~min}$ at $4^{\circ} \mathrm{C}$. 
A

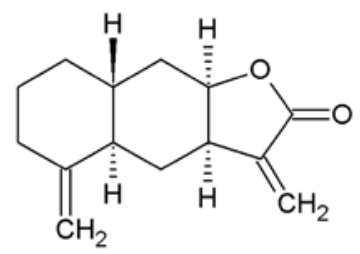

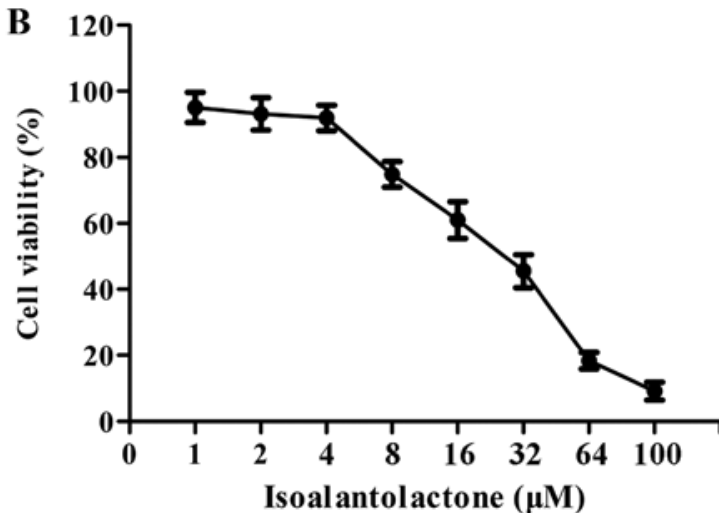

Figure 1. Effect of isoalantolactone on the viability of MDA-MB-231 cells. (A) Chemical structure of isoalantolactone. (B) MDA-MB-231 cells were treated with various concentrations of isoalantolactone, and cell viability was evaluated by MTT assay. All data are expressed as means \pm SD ( $=3$ ).

Western blot analysis. After whole-cell lysates were obtained, cytosolic and nuclear extracts were isolated, and equal quantities of protein were loaded onto $10 \%$ SDS-polyacrylamide gels for separation and then were transferred to a PVDF membrane blocked with 5\% skim milk. Subsequently, primary antibodies were used and then the membrane was washed for secondary antibody staining. Antibody binding was detected by enhanced chemiluminescence (Amersham Life Sciences, Amersham, UK). The quantification of proteins was analyzed by Image-Pro Plus (IPP) software.

Immunocytochemical analysis. Immunocytochemical assay was performed to determine the $\mathrm{NF}-\kappa \mathrm{B}$ p 65 protein level in the nucleus according to a previous method (22). MDA-MB-231 cells were grown on glass coverslips and incubated with isoalantolactone for $24 \mathrm{~h}$. Then, the cells were fixed with fresh $4 \%$ formaladehyde at room temperature for $90 \mathrm{~min}$ and washed twice with PBS. The cells were permeabilized with $0.1 \%$ Triton X-100 for $20 \mathrm{~min}$, and treated with 5\% bovine serum albumin (BSA; Santa Cruz Biotechnology Inc.). Primary anti-

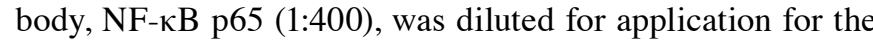
incubation of cells, followed by the secondary antibody. An Elivison two-step method was carried out for the immunohistochemical staining and image were captured using a DM2500 optical microscope. The positive expression was analyzed by IPP software.

Gelatin zymography assay. MDA-MB-231 cells were cultured with or without isoalantolactone $(1,2$, or $4 \mu \mathrm{M})$ in serum-free media for $24 \mathrm{~h}$, and then the supernatants were collected. The activities of MMP-2 and MMP-9 were estimated by gelatin zymography assay as previously described (23). Briefly, conditioned medium was electrophoresed on a $8 \%$ SDS-PAGE gel containing $0.1 \%$ gelatin. The gels were washed with $2.5 \%$ (v/v) Triton X-100 at room temperature, and then incubated in reaction buffer [50 mM Tris- $\mathrm{Cl}$ (pH 7.6), $10 \mathrm{mM} \mathrm{CaCl}_{2}$, $200 \mathrm{mM} \mathrm{NaCl}]$ at $37^{\circ} \mathrm{C}$ overnight to digest the gelatin. Finally, enzyme-digested regions were observed as clear bands against a blue background. Negatively stained bands were considered as the zones of enzymatic activity.

Luciferase reporter gene assay. The effect of isoalantolactone on $\mathrm{NF}-\kappa \mathrm{B}$-dependent reporter gene transcription was analyzed by NF- $\mathrm{B}$-luciferase assay. MDA-MB-231 cells $\left(5 \times 10^{5}\right.$ cells/well $)$ were plated in 6 -well plates and transiently transfected by the Liposome 2000 method with the pNF- $\mathrm{BB}-\mathrm{luc}$ plasmid reporter gene $(0.5 \mu \mathrm{g}$; Beyotime) and $\beta$-galactosidase (90 ng). After transfection for $24 \mathrm{~h}$, the cells were treated with isoalantolactone $(1,2$ or $4 \mu \mathrm{M})$ for an additional $24 \mathrm{~h}$. Cells were harvested for measuring $\beta$-galactosidase activity and luciferase activity. Relative luciferase activity was normalized to the $\beta$-galactosidase value to correct transfection efficacy.

Statistical analysis. All data in this study were taken from three independent experiments and are expressed as means \pm standard deviation (SD). The statistical significance was analyzed using the one-way analysis of variance (ANOVA) with the Statistical Package for the Social Sciences (SPSS, 13.0) software. Differences were considered significant at $\mathrm{p}<0.05$.

\section{Results}

Effect of isoalantolactone on the viability of the MDA-MB-231 cells. The effect of isoalantolactone on the cell viability of the MDA-MB-231 cells was determined by MTT assay. MDA-MB-231 cells were treated with isoalantolactone for $24 \mathrm{~h}$ at various concentrations $(1-100 \mu \mathrm{M})$. As depicted in Fig. 1B, isoalantolactone did not show any cytotoxic effect on the MDA-MB-231 cells at a dose $<4 \mu \mathrm{M}$. Therefore, we chose 1 , 2 and $4 \mu \mathrm{M}$ of isoalantolactone in the following experiments.

Isoalantolactone suppresses the adhesion, migration and invasion of $M D A-M B-231$ cells in vitro. We examined the effect of isoalantolactone on the adhesion ability of the MDA-MB-231 cells by MTT assay. As shown in Fig. 2A, the number of adhesive cells was markedly decreased following the treatment of isoalantolactone in a concentration-dependent manner, when compared with the blank control. This result indicated that isoalantolactone suppressed the invasiveness of the MDA-MB-231 cells by decreasing cell adhesion.

To investigate the effect of isoalantolactone on MDA-MB-231 cell migration, wound-healing assay was performed. The closure rate of MDA-MB-231 cells was determined by IPP software. As depicted in Fig. 2B and C, the MDA-MB-231 cells covered most of the wound area after a 24-h incubation in the control blank group. The wound closure 
A

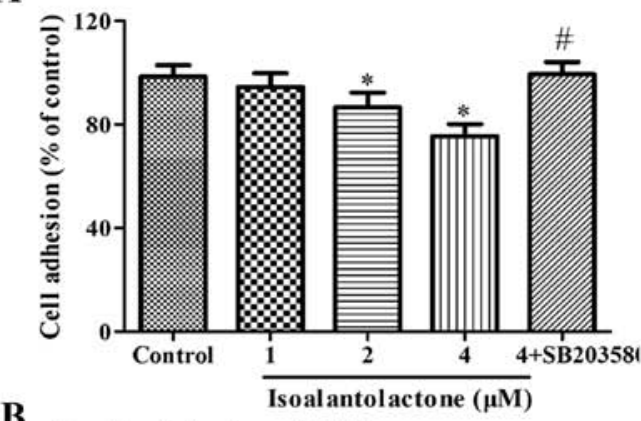

B

Isoalantolactone $(\mu \mathrm{M})$
C

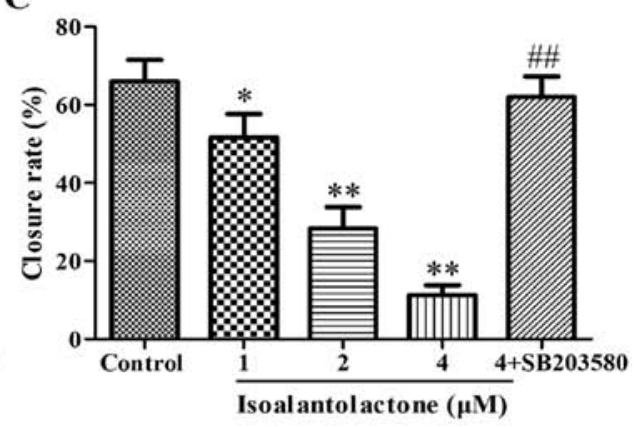

2

4

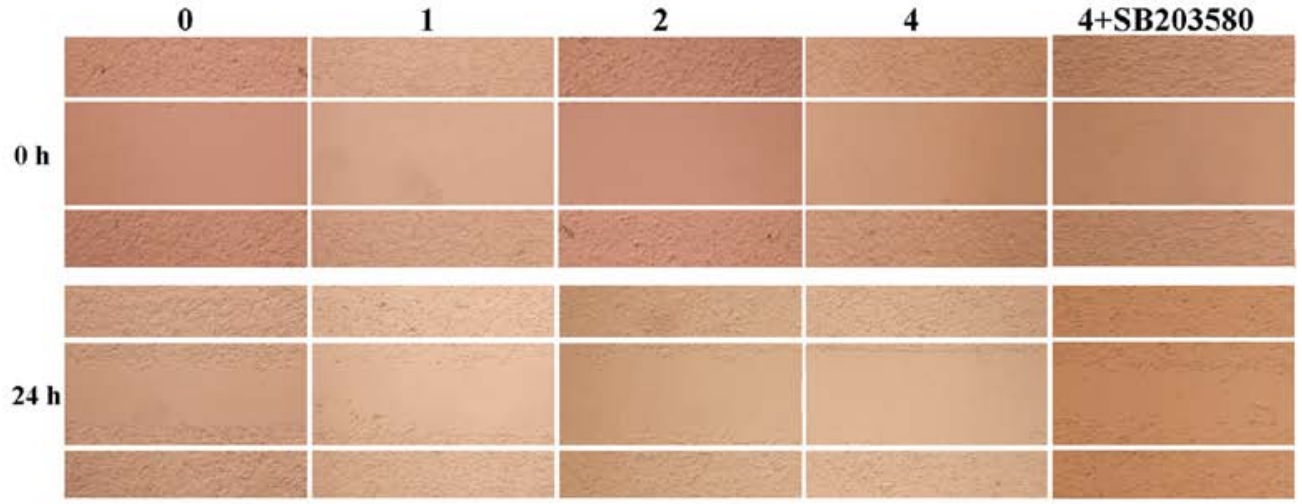

D

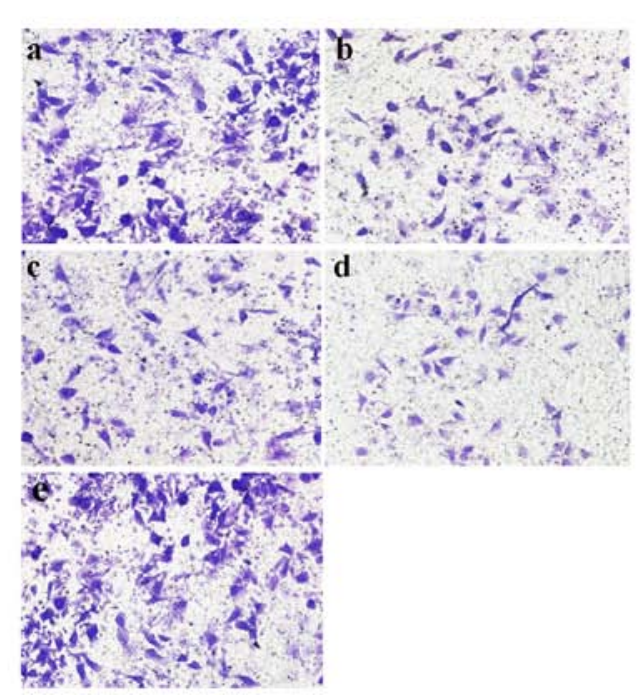

$\mathbf{E}$

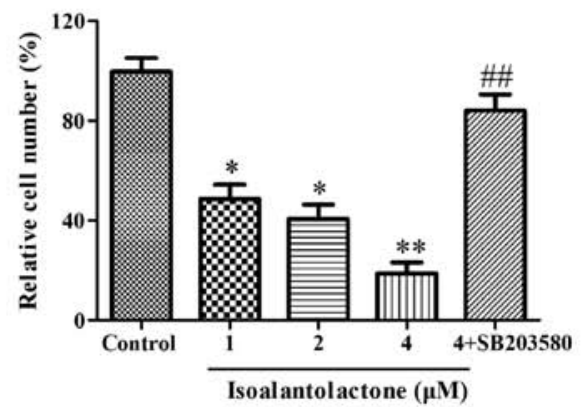

Figure 2. Effect of isoalantolactone on the adhesion, migration and invasion of MDA-MB-231 cells. (A) MTT assay of the adhesion of MDA-MB-231 cells. (B) Wound-healing assay for cell migration. (C) The closure rate was analyzed by IPP software. (D) MDA-MB-231 cells were inoculated in Matrigel-coated Transwell chambers cultured with isoalantolactone to determine cell invasion: (a) control, (b) isoalantolactone $1 \mu \mathrm{M}$, (c) isoalantolactone $2 \mu \mathrm{M}$, (d) isoalantolactone $4 \mu \mathrm{M}$, (e) isoalantolactone $4 \mu \mathrm{M}+\mathrm{SB} 203580$. (E) Optical density of the permeated cells. Data are expressed as means $\pm \mathrm{SD}(\mathrm{n}=3)$. ${ }^{*} \mathrm{p}<0.05,{ }^{* *} \mathrm{p}<0.01$, isoalantolactone vs. control; ${ }^{\#} \mathrm{p}<0.05,{ }^{\# \#} \mathrm{p}<0.01$, isoalantolactone $4 \mu \mathrm{M}+\mathrm{SB} 203580$ vs. isoalantolactone $4 \mu \mathrm{M}$.

rates of the groups treated with isoalantolactone at 1,2 and $4 \mu \mathrm{M}$ were $51.67 \pm 6.03,28.33 \pm 5.51$ and $11.33 \pm 2.52 \%$, respectively. These closure rates were all significantly smaller than the rate noted in the untreated group. Subsequently, we estimated the invasive ability of the MDA-MB-231 cells incubated in the presence or absence of isoalantolactone by the Transwell chamber assay. As shown in Fig. 2D and E, isoalantolactone inhibited the invasion of MDA-MB-231 cells in a concentration-dependent manner. To confirm the role of p38 MAPK in the inhibition of isoalantolactone on MDA-MB-231 cells, we added p38 MAPK $(4 \mu \mathrm{M})$ to cells with isoalantolactone $(4 \mu \mathrm{M})$. Then, the inhibiton of isoalantolactone on adhesion, migration and invasion was significantly reversed $(\mathrm{p}<0.05$, $\mathrm{p}<0.01)$. Overall, these results indicated that isoalantolactone decreased the adhesive, migratory, and invasive ability of highly metastatic MDA-MB-231 cells in vitro through the $\mathrm{p} 38$ MAPK signaling pathway.

Isoalantolactone downregulates the proteolytic activities and expression of MMP-2 and MMP-9 in MDA-MB-231 cells. To investigate the anti-invasive mechanism of isoalantolactone, the activity, protein expression and mRNA levels of MMP-2 and MMP-9 in the isoalantolactone-treated MDA-MB-231 cells were determined. As shown in Fig. 3, the activity as well 
A

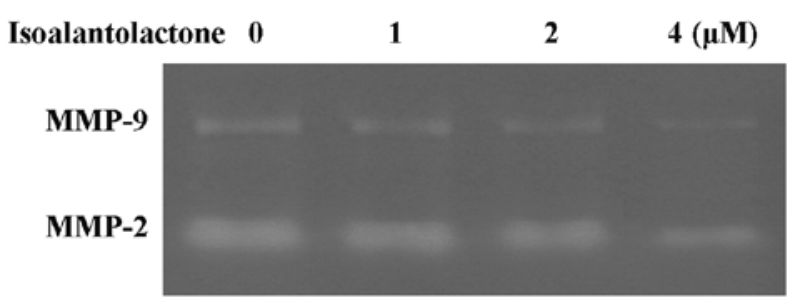

C

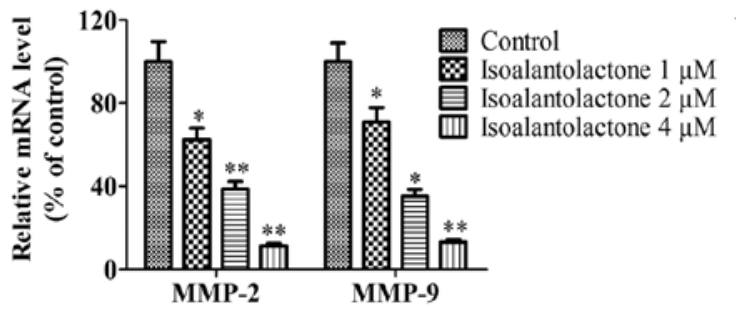

E

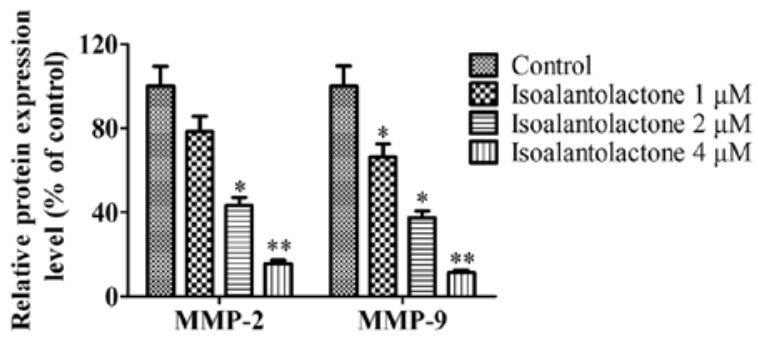

B

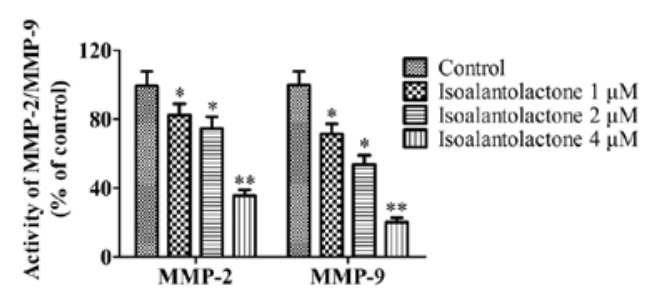

D

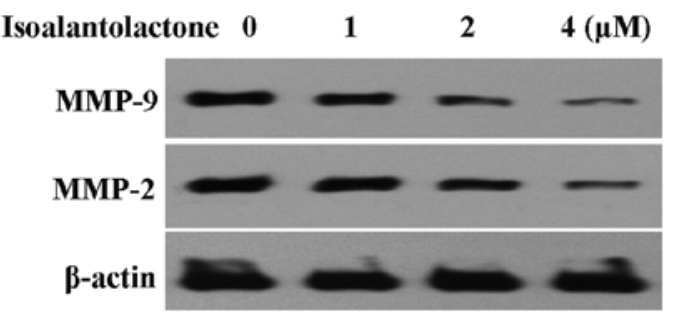

Figure 3. Isoalantolactone decreases the activity and expression of MMP-2 and MMP-x9 in MDA-MB-231 cells. (A and B) The activity of MMP-2 and MMP-9 was estimated by gelatin zymography. (C) The mRNA levels of MMP-2 and MMP-9 were estimated by real-time PCR. (D) The expression levels of MMP-2 and MMP-9 proteins were estimated by western blotting assay. (E) Quantification of MMP-2 and MMP-9 proteins by IPP software. Data are presented as means $\pm \mathrm{SD}(\mathrm{n}=3) .{ }^{*} \mathrm{p}<0.05,{ }^{* *} \mathrm{p}<0.01$, isoalantolactone vs. control.

as the protein and mRNA levels of MMP-2 and MMP-9 in the isoalantolactone-treated cells were downregulated in a concentration-dependent manner. These results indicated that isoalantolactone inhibited the invasion ability of MDA-MB-231 cells via suppression of the activity and expression of MMP-2 and MMP-9.

Isoalantolactone suppresses p38 MAPK activity. MAPK superfamily members, including p38, ERK and JNK, play important roles in cell invasion (24). To demonstrate whether isoalantolactone inhibits invasion by regulating the MAPK signaling pathway, we determined the protein expression of phosphorylated p38, ERK, JNK in the isoalantolactone-treated MDA-MB-231 cells. Western blotting indicated that isoalantolactone downregulated the protein expression of $\mathrm{p}-\mathrm{p} 38$ MAPK in a concentration-dependent manner with only a slight effect on the protein expression of p-ERK $1 / 2$ and p-JNK1/2 (Fig. 4A and B). The total protein levels were not affected. The observations revealed that isoalantolactone specifically suppressed p38 MAPK activity.

To explore the possible functional relationship between p38 MAPK and MMP-2 and MMP-9, MDA-MB-231 cells were treated with SB203580 (p38 MAPK-specific inhibitor) with or without isoalantolactone for $24 \mathrm{~h}$. Western blot analysis indicated that the inhibitor decreased the protein expression of MMP-2 and MMP-9 that was increased by isoalantolactone (Fig. 4C and D). The results indicated that isoalantolactone downregulated the expression of MMP-2 and MMP-9 proteins and in vitro invasion via blocking the activation of p38 MAPK.

Isoalantolactone inhibits $N F-\kappa B$ p 65 activation in the $M D A-M B-231$ cells. To demonstrate the involvement of the $\mathrm{NF}-\kappa \mathrm{B}$ pathway, the effect of isoalantolactone on NF- $\mathrm{B}$ p65 protein expression in the MDA-MB-231 cells was determined using western blot analysis. As shown in Fig. 5A and B, isoalantolactone markedly blocked the translocation of NF- $\kappa \mathrm{B}$ p65 from the cytoplasm into the nucleus. In addition, immunocytochemical analysis was performed to clarify the translocation

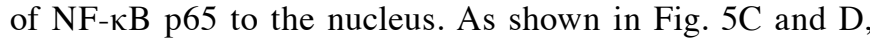
isoalantolactone inhibited the level of $N F-\kappa B$ p 65 protein in the nucleus in a dose-dependent manner.

Effect of isoalantolactone on $N F-\kappa B$-mediated transcriptional activity. The effect of isoalantolactone on NF- $\kappa \mathrm{B}-$ mediated transcriptional activity was assessed. As shown in Fig. 6A, isoalantolactone significantly reduced luciferase activity compared with that in the control cells in a dose-dependent manner. To demonstrate the essential role of $\mathrm{NF}-\kappa \mathrm{B}$ signaling in the inhibitory effect of isoalantolactone on invasion, MDA-MB-231 cells after transfection were treated with $4 \mu \mathrm{M}$ isoalantolactone. The result indicated that isoalantolactone 


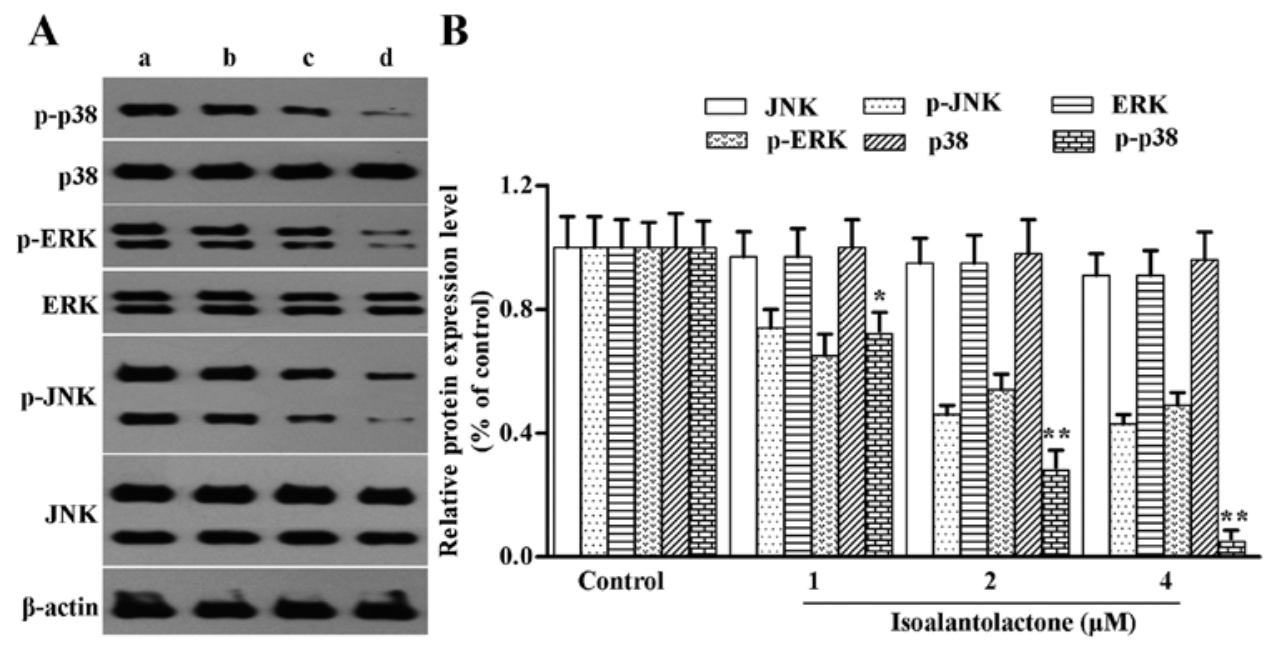

C

$\begin{array}{rllll}\text { SB203580 } & - & + & - & + \\ \text { Isoalantolacone } & - & - & + & + \\ \text { MMP-9 } & 0 & - & - & - \\ \text { MMP-2 } & & - & - & - \\ \beta \text {-actin } & & & \end{array}$

D

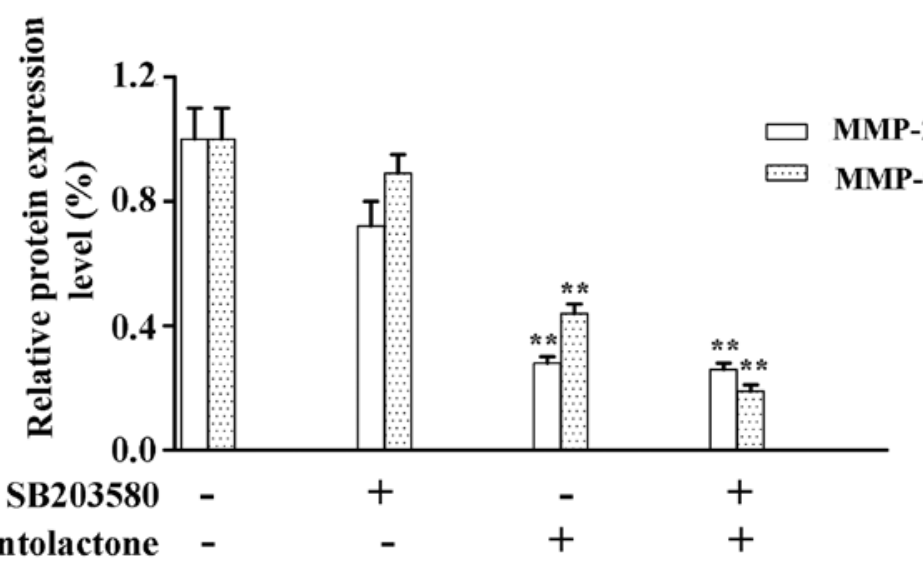

Figure 4. p38 MAPK is associated with isoalantolactone-suppressed MMP-2 and MMP-9 expression and in vitro invasion. (A and B) The expression of JNK1/2, p-JNK1/2, ERK1/2, p-ERK1/2, p38 MAPK, p-p38 MAPK proteins in the MDA-MB-231 cells after treatment with isoalantolactone for $24 \mathrm{~h}$ by western blot assay. (C and D) Expression of MMP-2 and MMP-9 proteins in cells after treatment with isoalantolactone or SB203580 or together for $24 \mathrm{~h}$, by western blot assay. Data are presented as means $\pm \operatorname{SD}(n=3) .{ }^{*} \mathrm{p}<0.05,{ }^{* *} \mathrm{p}<0.01$, isoalantolactone vs. control.

inhibited cell invasion (37\% decrease, Fig. 6B), which was weaker than the inhibitory effect of isoalantolactone on the non-transfected cells (Fig. 2D and E).

\section{Discussion}

Metastasis is the primary cause of the cancer-related high mortality rate worldwide $(25,26)$. Therefore, the search for agents which can be employed to effectively inhibit cancer metastasis is urgent (27). Due to drug resistance and toxic side-effects of current chemotherapy, herbal medicine has attracted the attention of scientists. Isoalantolactone is a natural sesquiterpene lactone extracted from Inula helenium. Recently published studies have demonstrated that isoalantolactone possesses anticancer activity against osteosarcoma U2OS, UM-SCC-10A and SGC-7901 cells $(13,16,18)$. In the present study, we found that isoalantolactone potently suppressed cell adhesion, migration and invasion at concentrations with no effect on cell viability to exert its anticancer effect (Figs. 1 and 2). Our study suggests that isoalantolactone may be a promising candidate agent against the metastasis of breast cancer.

MMPs, a family of well-characterized structurally related zinc-dependent proteases, play a pivotal role in tissue remodeling (28-30). Among the MMP members, MMP-2 and MMP-9 are commonly related to tumor migration, invasion, and metastasis in various human cancers, due to their capacities to degrade type IV collagen which is an important component of the basement membrane, leading to increased cell motility (31). Previous studies have confirmed that high levels and activation of MMP-2 and MMP-9 significantly enhance cancer cell invasion (32). Our findings indicated that isoalantolactone downregulated the activity and expression of MMP-2 and MMP-9 at the protein and mRNA levels in 
A

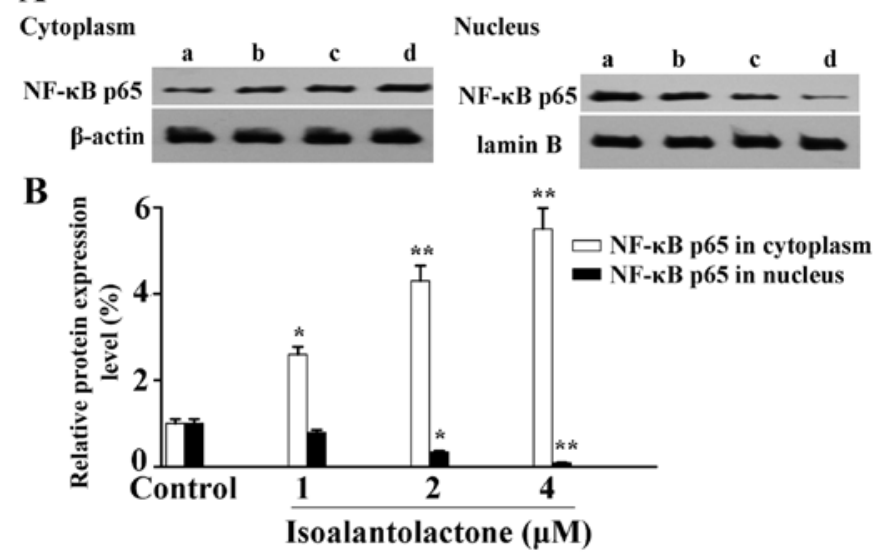

C

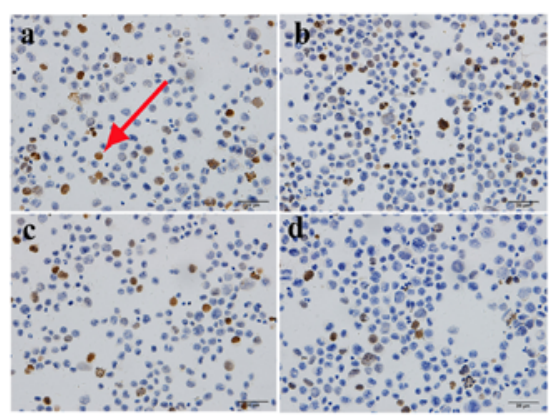

D

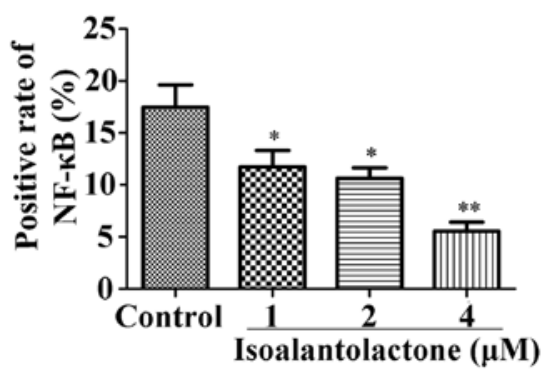

Figure 5. Effect of isoalantolactone on NF- $\mathrm{B}$ p65 activation in the MDA-MB-231 cells. (A) The NF- $\kappa \mathrm{B}$ p65 protein expression in the cytoplasm and nucleus by western blot assay. (B) Quantification of NF- $\kappa$ B p65 protein expression in the cytoplasm and nucleus by IPP software. (C) The $\mathrm{NF}-\kappa \mathrm{B}$ p 65 protein expression in the nucleus by immunocytochemical analysis: (a) control, (b) isoalantolactone $1 \mu \mathrm{M}$, (c) isoalantolactone $2 \mu \mathrm{M}$, (d) isoalantolactone $4 \mu \mathrm{M}$. The red arrow represents the brown-stained positive expression. (D) The relative positive expression of $\mathrm{NF}-\kappa \mathrm{B}$ p65 protein in the immunocytochemical analysis. Data are presented as means $\pm \mathrm{SD}(\mathrm{n}=3)$. ${ }^{*} \mathrm{p}<0.05,{ }^{* *} \mathrm{p}<0.01$, isoalantolactone vs. control.

a dose-dependent manner in MDA-MB-231 cells (Fig. 3), suggesting that isoalantolactone inhibited cancer cell invasion via a decrease in MMP-2 and MMP-9, thus resulting in failure of tissue remodeling via the ECM.

The MAPK-mediated signaling pathway is closely associated with cell invasion and metastasis during the development and progression of tumors $(33,34)$. It was recently reported that the MAPK signaling pathway is involved in the modulation of MMP-2 and MMP-9 levels in cancer cells (35). In the present study, we found that isoalantolactone decreased the levels of p-p38 MAPK, but did not reduce the expression of p-ERK1/2 or p-JNK1/2 (Fig. 4A and B). Additionally, SB203580 (a specific inhibitor of p38 MAPK) reduced the expression of MMP-2 and MMP-9 proteins and suppressed MDA-MB-231 cell invasion to the same degree as did
A

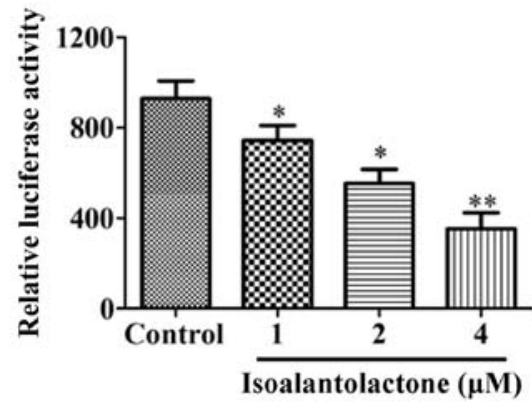

B

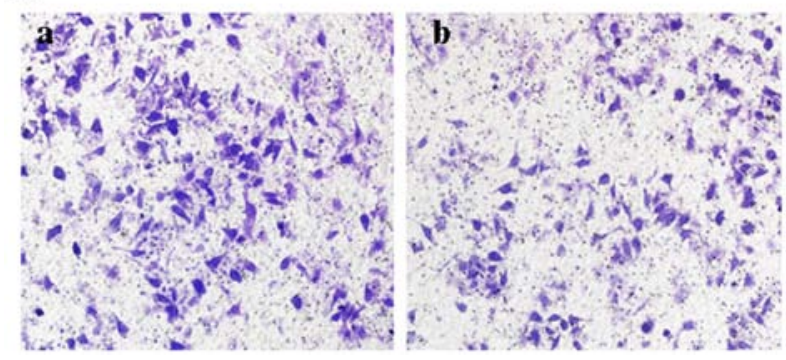

Figure 6. Effect of isoalantolactone on the $\mathrm{NF}-\kappa \mathrm{B}$ signaling pathway. (A) Effect of isoalantolactone on NF- $\mathrm{B}$-dependent reporter gene transcription ability. (B) Effect of isoalantolactone on invasive ability in the transfected cells. Data are presented as means $\pm \mathrm{SD}(\mathrm{n}=3) .{ }^{*} \mathrm{p}<0.05,{ }^{* *} \mathrm{p}<0.01$, isoalantolactone vs. control.

isoalantolactone (Fig. 4C-E). The results were in line with previous studies, which demonstrated that $\mathrm{p} 38$ MAPK activity was needed for 12- $O$-tetradecanoylphorbol-13-acetate (TPA)-, hepatitis $\mathrm{C}$ virus (HCV)-, HOXA10 overexpression-induced increase of MMP in cancer cells $(9,36,37)$. Thus, isoalantolactone inhibits the invasion of MDA-MB-231 cells via blocking the activity of $\mathrm{p} 38$ MAPK.

It was reported that activation of the p38 MAPK pathway activates NF- $\kappa B$, which promotes NF- $\kappa B$ translocation to the nucleus and then regulates NF- $\kappa B$-dependent MMP transcription (38). Activated NF- $\kappa$ B by p38 MAPK induced the invasion of prostate cancer cells by inhibiting MMP-9 expression (39). Our results indicated that isoalantolactone inhibited the translocation of NF- $\mathrm{\kappa B}$ p65 to the nucleus (Fig. 5). Therefore, isoalantolactone decreased the level of MMPs and had an antiinvasive effect.

In summary, the present study demonstrated for the first time that isoalantolactone treatment resulted in the inhibition of adhesion, migration, and invasion of highly metastatic MDA-MB-231 breast cancer cells in vitro. Isoalantolactone reduced the level and activation of MMP-2 and MMP-9 by blocking the p38 MAPK signaling pathway and NF- $\mathrm{kB}$ p65 transcriptional activity. These results provide experimental evidence for further development of isoalantolactone as a potent agent against MDA-MB-231 cells and might be used as a promising chemopreventive agent for the treatment of breast cancer.

\section{Acknowledgements}

The present study was supported by the National Natural Science Foundation of China (no. 81403121). 


\section{References}

1. Carvalho I, Milanezi F, Martins A, Reis RM and Schmitt F: Overexpression of platelet-derived growth factor receptor alpha in breast cancer is associated with tumour progression. Breast Cancer Res 7: R788-R795, 2005

2. Côme C, Laine A, Chanrion M, Edgren H, Mattila E, Liu X, Jonkers J, Ivaska J, Isola J, Darbon JM, et al: CIP2A is associated with human breast cancer aggressivity. Clin Cancer Res 15 5092-5100, 2009.

3. Cheng X, Gu J, Zhang M, Yuan J, Zhao B, Jiang J and Jia X: Astragaloside IV inhibits migration and invasion in human lung cancer A549 cells via regulating PKC- $\alpha$-ERK1/2-NF- $\kappa$ B pathway. Int Immunopharmacol 23: 304-313, 2014.

4. Mohammad MA, Zeeneldin AA, Abd Elmageed ZY, Khalil EH, Mahdy SM, Sharada HM, Sharawy SK and Abdel-Wahab AH: Clinical relevance of cyclooxygenase-2 and matrix metalloproteinases (MMP-2 and MT1-MMP) in human breast cancer tissue. Mol Cell Biochem 366: 269-275, 2012.

5. Kim TD, Song KS, Li G, Choi H, Park HD, Lim K, Hwang BD and Yoon WH: Activity and expression of urokinase-type plasminogen activator and matrix metalloproteinases in human colorectal cancer. BMC Cancer 6: 211, 2006.

6. Bodey B, Bodey B Jr, Gröger AM, Siegel SE and Kaiser HE: Invasion and metastasis: The expression and significance of matrix metalloproteinases in carcinomas of the lung. In Vivo 15: 175-180, 2001.

7. Dhillon AS, Hagan S, Rath O and Kolch W: MAP kinase signalling pathways in cancer. Oncogene 26: 3279-3290, 2007.

8. Trusolino L and Comoglio PM: Scatter-factor and semaphorin receptors: Cell signalling for invasive growth. Nat Rev Cancer 2: 289-300, 2002.

9. Noh EM, Park YJ, Kim JM, Kim MS, Kim HR, Song HK, Hong OY, So HS, Yang SH, Kim JS, et al: Fisetin regulates TPA-induced breast cell invasion by suppressing matrix metalloproteinase-9 activation via the PKC/ROS/MAPK pathways. Eur J Pharmacol 764: 79-86, 2015.

10. Xia P, Zhang R and Ge G: C/EBP $\beta$ mediates TNF- $\alpha$-induced cancer cell migration by inducing MMP expression dependent on p38 MAPK. J Cell Biochem 116: 2766-2777, 2015.

11. Liu ZL, Liu Q, Xiao B, Zhou J, Zhang JG and Li Y: The vascular protective properties of kinsenoside isolated from Anoectochilus roxburghii under high glucose condition. Fitoterapia 86: 163-170, 2013.

12. Schmidt TJ,BrunR, WilluhnG and KhalidSA: Anti-trypanosomal activity of helenalin and some structurally related sesquiterpene lactones. Planta Med 68: 750-751, 2002.

13. Di W, Khan M, Rasul A, Sun M, Sui Y, Zhong L, Yang L, Zhu Q, Feng L and Ma T: Isoalantolactone inhibits constitutive $\mathrm{NF}-\kappa \mathrm{B}$ activation and induces reactive oxygen species-mediated apoptosis in osteosarcoma U2OS cells through mitochondrial dysfunction. Oncol Rep 32: 1585-1593, 2014.

14. Stojanović-Radić Z, Comić Lj, Radulović N, Blagojević P, Denić M, Miltojević A, Rajković J and Mihajilov-Krstev T: Antistaphylococcal activity of Inula helenium L. root essential oil: Eudesmane sesquiterpene lactones induce cell membrane damage. Eur J Clin Microbiol Infect Dis 31: 1015-1025, 2012.

15. Kumar A, Kumar S, Kumar D and Agnihotri VK: UPLC/MS/MS method for quantification and cytotoxic activity of sesquiterpene lactones isolated from Saussurea lappa. J Ethnopharmacol 155: 1393-1397, 2014.

16. Wu M, Zhang H, Hu J, Weng Z, Li C, Li H, Zhao Y, Mei X, Ren F and Li L: Isoalantolactone inhibits UM-SCC-10A cell growth via cell cycle arrest and apoptosis induction. PLoS One 8: e76000, 2013.

17. Rasul A, Di J, Millimouno FM, Malhi M, Tsuji I, Ali M, Li J and Li X: Reactive oxygen species mediate isoalantolactone-induced apoptosis in human prostate cancer cells. Molecules 18 9382-9396, 2013.

18. Rasul A, Khan M, Yu B, Ali M, Bo YJ, Yang H and Ma T: Isoalantolactone, a sesquiterpene lactone, induces apoptosis in SGC-7901 cells via mitochondrial and phosphatidylinositol 3-kinase/Akt signaling pathways. Arch Pharm Res 36: 1262-1269, 2013.

19. Zhang C, Chi YL, Wang PY, Wang YQ, Zhang YX, Deng J, Lv CJ and Xie SY: miR-511 and miR-1297 inhibit human lung adenocarcinoma cell proliferation by targeting oncogene TRIB2. PLoS One 7: e46090, 2012.
20. Wu W, He X, Kong J and Ye B: mir-373 affects human lung cancer cells' growth and its E-cadherin expression. Oncol Res 20: 163-170, 2012.

21. Albini A, Benelli R, Noonan DM and Brigati C: The 'chemoinvasion assay': A tool to study tumor and endothelial cell invasion of basement membranes. Int J Dev Biol 48: 563-571, 2004.

22. Feng L, Zhu MM, Zhang MH, Wang RS, Tan XB, Song J, Ding SM, Jia XB and Hu SY: Protection of glycyrrhizic acid against AGEs-induced endothelial dysfunction through inhibiting RAGE/NF- $\mathrm{B}$ pathway activation in human umbilical vein endothelial cells. J Ethnopharmacol 148: 27-36, 2013.

23. Wang L, Ling Y, Chen Y, Li CL, Feng F, You QD, Lu N and Guo QL: Flavonoid baicalein suppresses adhesion, migration and invasion of MDA-MB-231 human breast cancer cells. Cancer Lett 297: 42-48, 2010.

24. Reddy KB, Nabha SM and Atanaskova N: Role of MAP kinase in tumor progression and invasion. Cancer Metastasis Rev 22: 395-403, 2003

25. Wang S, Liu Q, Zhang Y, Liu K, Yu P, Liu K, Luan J, Duan H, Lu Z, Wang F, et al: Suppression of growth, migration and invasion of highly-metastatic human breast cancer cells by berbamine and its molecular mechanisms of action. Mol Cancer 8: 81, 2009.

26. Xu E, Zhao J, Ma J, Wang C, Zhang C, Jiang H, Cheng J, Gao R and Zhou X: miR-146b-5p promotes invasion and metastasis contributing to chemoresistance in osteosarcoma by targeting zinc and ring finger 3. Oncol Rep 35: 275-283, 2016.

27. Jemal A, Siegel R, Ward E, Hao Y, Xu J, Murray T and Thun MJ: Cancer statistics, 2008. CA Cancer J Clin 58: 71-96, 2008.

28. McCawley LJ and Matrisian LM: Matrix metalloproteinases: Multifunctional contributors to tumor progression. Mol Med Today 6: 149-156, 2000.

29. Yan C and Boyd DD: Regulation of matrix metalloproteinase gene expression. J Cell Physiol 211: 19-26, 2007.

30. Kim JH, Yang YI, Ahn JH, Lee JG, Lee KT and Choi JH: Deer (Cervus elaphus) antler extract suppresses adhesion and migration of endometriotic cells and regulates MMP-2 and MMP-9 expression. J Ethnopharmacol 140: 391-397, 2012.

31. Yang SF, Chen MK, Hsieh YS, Yang JS, Zavras AI, Hsieh YH, Su SC, Kao TY, Chen PN and Chu SC: Antimetastatic effects of Terminalia catappa L. on oral cancer via a down-regulation of metastasis-associated proteases. Food Chem Toxicol 48: 1052-1058, 2010.

32. Puricelli L, Dell'Aica I, Sartor L, Garbisa S and Caniato R: Preliminary evaluation of inhibition of matrix-metalloprotease MMP-2 and MMP-9 by Passiflora edulis and $P$. foetida aqueous extracts. Fitoterapia 74: 302-304, 2003.

33. Niu J, Huang Y and Zhang L: CXCR4 silencing inhibits invasion and migration of human laryngeal cancer Hep-2 cells. Int J Clin Exp Pathol 8: 6255-6261, 2015.

34. Zhan Y, Zhang H, Liu R, Wang W, Qi J and Zhang Y: Eupolyphaga sinensis Walker ethanol extract suppresses cell growth and invasion in human breast cancer cells. Integr Cancer Ther 15: 102-112, 2016.

35. Chen Y, Zheng L, Liu J, Zhou Z, Cao X, Lv X and Chen F: Shikonin inhibits prostate cancer cells metastasis by reducing matrix metalloproteinase-2/-9 expression via AKT/mTOR and ROS/ERK1/2 pathways. Int Immunopharmacol 21: 447-455, 2014.

36. Lu L, Zhang Q, Wu K, Chen X, Zheng Y, Zhu C and Wu J: Hepatitis $C$ virus NS3 protein enhances cancer cell invasion by activating matrix metalloproteinase- 9 and cyclooxygenase- 2 through ERK/p38/NF- $\mathrm{B}$ signal cascade. Cancer Lett 356: 470-478, 2015.

37. Cui XP, Qin CK, Zhang ZH, Su ZX, Liu X, Wang SK and Tian XS: HOXA10 promotes cell invasion and MMP-3 expression via TGF 32 -mediated activation of the $\mathrm{p} 38$ MAPK pathway in pancreatic cancer cells. Dig Dis Sci 59: 1442-1451, 2014.

38. Kim D, Kim S, Koh H, Yoon SO, Chung AS, Cho KS and Chung J: $\mathrm{Akt} / \mathrm{PKB}$ promotes cancer cell invasion via increased motility and metalloproteinase production. FASEB J 15: 1953-1962, 2001.

39. Xu P, Cai F, Liu X and Guo L: Sesamin inhibits lipopolysaccharide-induced proliferation and invasion through the p38-MAPK and NF- $\mathrm{KB}$ signaling pathways in prostate cancer cells. Oncol Rep 33: 3117-3123, 2015. 